\title{
The possibilities and limits of clinical epidemiology
}

\author{
Departamento de Medicina Preventiva da FMUSP
}

The production of scientific knowledge and the development of modern technologies have had an impressive growth in recent years, as can be observed by the rapid and profound changes they promote in pre-existing knowledge. New products introduce radical changes in most technical-scientific areas, and the health sector constitutes one of the most sensitive areas. Modern biotechnology, Informatics, New materials and Chemistry are some examples of technological areas whose development has contributed to the "technological revolution", characteristic of our times. At the same time, knowledge produced by molecular biology has promoted significant changes in the way we understand the human nature and its organization.

In this setting, Clinical Medicine, like other disciplines, has suffered enormous changes, whose effects can be measured by the incorporation of modern diagnostic technologies the renewal of therapeutic options. While the first have been improved in their exactness and capacity of detection, leading therefore better diagnostic abilities, the later offer new perspectives, until recently unatainable, of control or cure of several problems and diseases, changing, quite frequently, actions and concepts until firmly established in Medicine.

This new situation has, on one hand, facilitated clinical reasoning, on the other one requires the adoption of parameters and procedures in a certain sense ignored by the traditional clinic investigations. Based on the case observations, which allows for considering "each case as an unique event", recent tendencies have made use of other techniques for scientific analysis, using epidemiological methodology as a scientific model. These implies in incorporating quantification (whose exponential growth curves anchor on the continuous development of computer techniques) as an auxiliary data interpretation technique based on probability concepts (Silva) as well as better designs for interpretations, thus strenghtening the "clinical trials".

This positive interaction between epidemiological methodology and clinical practice led to the growth of a new scientific discipline, Clinical Epidemiology, ultimate reason for this symposium. The object of intense debate among clinical-epidemiologists and epidemiologists (Sackett, Feinstein, Fletcher, Holland, Terris, among others) while presenting itself as the scientific method in Clinical Medicine, it has led to new possibilities of development in knowledge about diseases causation and medical intervention. Causal and explanatory questions have gained considerable impulse, giving more rationality and efficacy to the clinical approach, as can be seen in the enormous production in national and international literature, about individual health problems and the stablishment of its risk factors (one of the central concepts of epidemiology) leading to more adequate medical prescriptions and striving for longer and with better quality life. However, a methodological problem remains, since there is no direct mediation between probability observations applicable to a group of individuals and the specific situation of each individual exposed to a certain factor or submitted to specific therapeutics.

In spite of all advances, contrasts persist, particularly in countries with economies similar to Brazil. The incorporation of the produced knowledge, very promising on the Clini$\mathrm{cal}$, on the individual level, is much slower when applicable to the populational level, epidemiology's central object - the individual in its collective dimension (Castellanos). If on the one hand this may be due to the deficiences in the health services structure, on the other hand may point to difficulties with the method, when used for different objects, leading to situations where the proposed interventions do not relate to the true reasons for existing health problems.

This last observation permits identifying the fact that part of the existing polemics, particularly between the "classical epidemiologists" and the "clinical epidemiologists" are based not so much on technical-scientific differences but on distinct conceptions about the uses and possibilities of existing knowledge leading to effective interventions on health problems. 